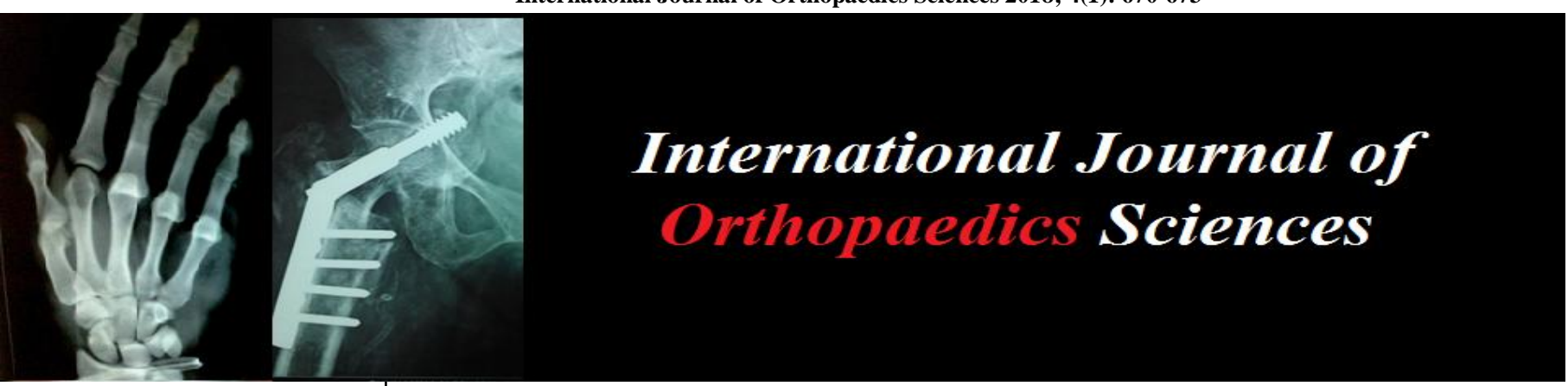

ISSN: $2395-1958$

IJOS 2018; 4(1): 670-673

(C) 2018 IJOS

www.orthopaper.com

Received: 16-11-2017

Accepted: 18-12-2017

Dr. D Vimal Raj

Associate Professor, Department of Orthopaedics, Indira Gandhi

Medical College \& Research

Institute, Puducherry, India

Dr. Marie Bernard Arokiaraj C Junior Resident, Department of Orthopaedics, Indira Gandhi Medical College \& Research Institute, Puducherry, India

Dr. Anish Anto Xavier Junior Resident, Department of Orthopaedics, Indira Gandhi Medical College \& Research Institute, Puducherry, India
Correspondence

DR. D Vimal Raj

Associate Professor, Department of Orthopaedics-Indira Gandhi

Medical College \& Research

Institute, Puducherry, India

\section{Functional and radiological outcome of intertrochanteric fracture treated with proximal femoral nail}

\author{
Dr. D Vimal Raj, Dr. Marie Bernard Arokiaraj C and Dr. Anish Anto \\ Xavier
}

DOI: https://doi.org/10.22271/ortho.2018.v4.i1j.98

\section{Abstract}

Intertrochanteric fractures are one of the most common fractures of the elderly due to simple fall but can also occur in younger age group after high energy trauma. Both male and female are equally affected irrespective of the mode of injury. The purpose of this study is to evaluate the functional and radiological outcome of intertrochanteric fracture treated with proximal femoral nail. The study was carried out with a total of 20 patients (male: 17, female: 3) treated with proximal femoral nail from the year 2015-2016. The inclusion criteria were patients with closed fractures of less than 3 weeks and age above 25 . These patients were evaluated clinically and radiologically at intervals of $4 \mathrm{wks}, 8 \mathrm{wks}, 12 \mathrm{wks}, 16 \mathrm{wks}$ and 20wks. The functional assessment was done using Harris Hip score. In our study majority were unstable fractures $(65 \%)$ and others were stable fractures $(35 \%) .50 \%$ of our total patients showed full union by 10 to 15 weeks and $35 \%$ showed full union by 16 to 20 weeks. We had $95 \%$ of the patients with good anatomical results and $50 \%$ of the patients with excellent functional results. PFN is safe, effective and well tolerated device useful in the treatment of all intertrochanteric fractures. Being a closed intramedullary procedure early mobilization and rehabilitation is possible with restoration of normal functions.

Keywords: Intertrochanteric, proximal femoral nailing, femur

\section{Introduction}

Intertrochanteric fractures are one of the most common fractures usually encountered in elderly due to simple fall and can also occur in younger age group after high energy trauma involving both direct and indirect forces. Direct forces act along the axis of the femur or directly over the greater trochanter to result in an intertrochanteric fracture. The proposed surgery for our study is Proximal Femoral Nailing. PFN is the latest intramedullary device designed by AO / ASIF in 1996, which has got two proximal screws for the neck and head and two distal locking screws. The main advantage is being a closed technique offers good reduction at the fracture site and provides sufficient strength to allow weight bearing even in unstable hip fractures. Proximal femoral nail which is an intramedullary device is proven to be biomechanically superior to DHS which is an extramedullary device in the treatment of intertrochanteric fractures.

\section{Materials and Methods}

The study was conducted with 25 patients with intertrochanteric fracture evaluated and admitted in the Department of Orthopaedics, Indira Gandhi Medical College \& Research Institute, Puducherry from 2015-2016. Patients were selected based on the inclusion criteria such as Patients with intertrochanteric fractures of less than 3 weeks, Closed fractures and age above 25 including both sex groups and the exclusion criteria such as Fractures of more than 3 weeks, Open fractures, Age below 25 years, Patient unfit for surgery and Patient already operated elsewhere. After the initial clinical evaluation of the patient, blood investigation and radiological work up done for pre-anaesthetic check-up. All the patients were planned for proximal femoral nailing patient and underwent surgery on elective basis after the consent. 
Modalities of surgery and the application of the proposed technique used for fixation of the implant was recorded. Postoperative activities such as medications, regular wound inspection, suture removal and Mobilization from day 1 with subsequent ambulation training initiated using a walker or crutches were also documented. Patients were advised for regular follow ups on OPD basis at 4,8,12,16,20 weeks post operatively. During these visits, patients were assessed both clinically and radiologically based on pain, hip movements, fracture union and complications using Harris Hip score.

\subsection{Operative Technique}

Under spinal or general anaesthesia patient was put supine on the fracture table. The contralateral leg was placed on a leg support. A closed reduction of the fracture under image intensifier control was carried out. An incision of 2.5 to $3 \mathrm{~cm}$ was made $2 \mathrm{~cm}$ above proximal end of the greater trochanter on the extension line of the femoral axis. The soft tissue were cut reached the trochanter tip and the entry point was identified by touch located at the junction of anterior two third and posterior two thirds of greater trochanter. The entry hole was made with the awl, the guide wire was passed through the hole along the femoral axis and the reduction was checked with $\mathrm{C}$ arm in two planes. Reaming of the proximal and distal two fragments were done using 10, I 1, 12, 13, 14 reamers. Nail of at least $2 \mathrm{~mm}$ less than that of the last reamer used was selected. After fixing into the jig using the AP view, the PFN was inserted by hand until the lagscrew holes are lined up within the centre of femoral neck. Later the guide sleeve was introduced through the jig and a $2 \mathrm{~cm}$ incision was made on the lateral aspect of the thigh. A mark was made on the femur and the trocar was removed. A $2.8 \mathrm{~mm}$ guide wire was inserted through the drill sleeve into the bone and both direction and position were checked under image intensifier in AP and lateral view. A position in the caudal area of the femoral head was selected so that both proximal screws can be inserted. In lateral view, the wire should be in the centre the femoral neck. The guide wire was inserted subchondrally or $5 \mathrm{~mm}$ away into the femoral head. After reaming over the guide wire, the appropriate caudal hip screw was introduced.Then the cranial hip screw was introduced just above the caudal hip screw using the jig. Both the distal locking screws were inserted to improve the stability and the wound is closed with 1-3 stitches.

\section{Results and Discussion}

\subsection{Results}

A Prospective study consisting of 20 patients who underwent closed reduction internal fixation with proximal Femoral nail was conducted to study the functional and anatomical outcome in relation to the type of surgical treatment. Age of involvement ranging from 25 to 70 years with male predominance of $85 \%$ and female predominance of $15 \%$ were noticed and most of the patients were slip and fall $(65 \%)$ and the rest were road traffic accidents (35\%). Majority were unstable fractures according to Boyd and Griffin (65\%) and the rest were stable fractures $(35 \%)$. Post operatively patients were mobilized on the second day with non-weight bearing crutch walking $(60 \%)$ and partial weight bearing $(45 \%)$ in the first five weeks later with full weight bearing in the first 10 to 14 weeks (55\%). 50\% of the patients showed full union by 10 to 15 weeks and $35 \%$ showed full union by 16 to 20 weeks, the mean time taken for full union was 16 weeks. The mean range of motion for hip was 101 degrees and the knee was 134 degrees, We had $95 \%$ of the patients with good anatomical results and $50 \%$ of the patients with excellent functional results.

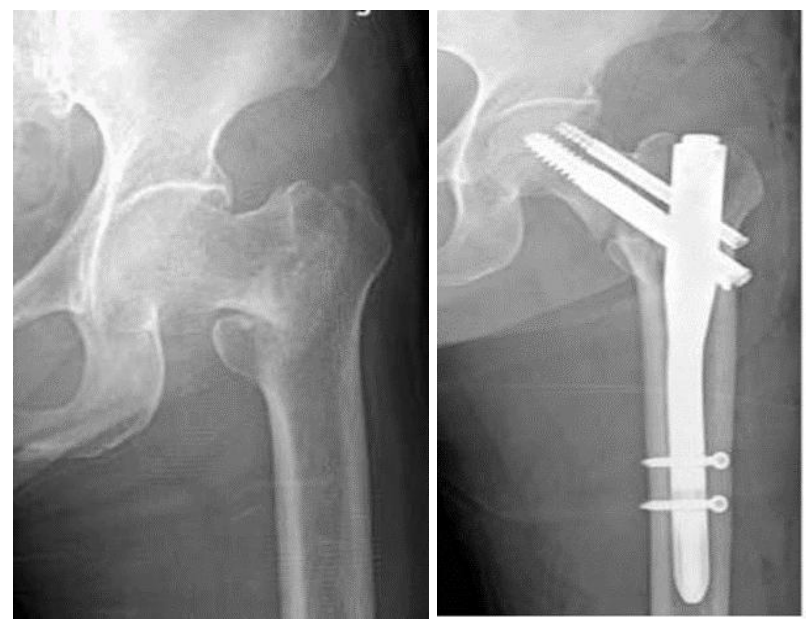

\subsection{Discussion}

The femur is the longest and strongest bone of the body and like all long bones consists of a shaft and two ends. It articulates at its upper end with the hipbone and at its lower end with both the patella and the tibia. The upper end of the femur comprises a head, a neck, a greater and a lesser trochanter. The neck of the femur is rather more than half a Sphere\& and is directed upwards, medially and slightly anteriorly. The neck is about $5 \mathrm{~cm}$ long, connects the head to the shaft. Anteriorly at the junction of the shaft and the neck is a rough bony ridge, the intertrochanteric line. It begins in a tubercle at the upper and medial part of the anterior surface of the greater trochanter and is directed inferomedially where it joins the spiral line, which becomes continuous with the medial lip of the linea aspera. Posteriorly a prominent ridge of bone, the intertronchanteric crest is a round protuberance called the quadrate tubercle. The greater trochanter is large and quadrangular laterally positioned and irregular. The upper posterior margin overhangs the trochanteric fossa. The greater trochanter provides insertion for most of the muscles of the gluteal region. The lesser trochanter is a conical eminence, which projects medially and backwards from the shaft at its junction with the lower and posterior part of the neck. It gives attachment to the psoas major on its summit and the iliacus at its base.

The description of adult vessels is based on the work of Trueta and Harnington (1953). Since the vascular pattern established during the phase of growth is not replaced at maturity, but persists throughout in life, the basic arrangement is one of an epiphyseal and metaphyseal circulation, even when the growth plate has disappeared outline the anastomotic arrangement around the upper femur. Corck described the blood supply to the proximal end of the femur, which he divided into three major groups. 1)An extracapsular arterial ring located at the base of the femoral neck. 2)Ascending cervical branch of the arterial ring on the surface of the femoral neck. 3)Arteries of the ligamentum teres. The extracapsular arterial ring is formed posteriorly by 
a large branch of medial femoral circumflex artery and anteriorly by branch from Ideral femoral circumflex artery. The ascending cervical branches of retinacular vessels ascend on the surface of the femoral neck in anterior, posterior, medial and lateral groups. The lateral vessels are most important. Their proximity to the surface of the femoral neck makes them vulnerable to injury in femoral neck fractures. Blood supply to the femur, like that of all tubular bones, is by the way of metaphyseal, periosteal and endosteal supply. The periosteal supply is related to the multiple muscle origins from the shaft to the femur. The nutrient arteries perforate the femoral shaft along the linea aspera. The arteries are derived from perforating branches of profounda femoris artery. The pertrochanteric and subtrochanteric area can be a site of stress concentration owing to the short radius of curvature at this site. When bone has insufficient opportunity to turnover and remodeling as in metabolic bone disease this may be a site of pathologic fracture. Muscular Forces: The upper end of the femur is surrounded by a mass of powerful muscles. Inclusion of muscles forces necessary during single leg support adds to the complexity of the problem and can increase the stress to much higher values. On the other hand, some muscles such as the tensor fascia lata, may act to partially neutralize bending forces under certain conditions. In a normal hip, the strong gluteal muscles abduct, and the powerful psoas flexes and rotates. These forces are balanced by the abductors and harmstrings. The same muscle forces act upon the fixation device after operation. These forces have been shown to generate high forces on the femoral head even when the patient is in bed, which in turn cause stresses in the subtrochanteric area as shown by Koch. Rydell has demonstrated that muscular pull for merely flexing of extending the hip in bed caused as much pressure on the femoral head as did slow walking with or without crutches. A pertrochanteric fracture is defined as one in which the main plane of bony separation passes through the greater trochanter obliquely downwards and inwards to or through the lesser trochanter Mechanism of Injury: Pertrochanteric fractures almost invariably occur as a result of a fall, involving both direct and indirect forces. Direct forces act along the axis of the femur or directly over the greater trochanter to result in an intertrochanteric fracture. Indirect forces, including pull of the ilipsoas muscle on the lesser trochanter and the abductors on the greater trochanter, have also been incriminated as a cause of fracture. Since this fracture is common in older age group, many of these fractures are of fatigue type in which the architecture of the bone is sufficiently inadequate that the fracture takes place with relatively normal activity, carefully taken history in many instances reveal that the patient had some minor mishap, such as turning round or possibly catching a heel, but the fracture often occurs before the fall. In younger individuals with stronger bones the fracture may result from a direct blow or twist in automobile accidents PFN for proximal femur is available to fit specific anatomic requirement and fracture type of proximal femur. Proximal locking is achieved through two $8 \mathrm{~mm}$ femoral neck screws. Proximal fixation delivers rotational stability.6* mediolateral implant angle for easy insertion. The anatomic nail design prevents intramedullary stresses and thus secondary fractures at the end of the nail. PFN nail sizes are 9, 10, 11 , \&amp; $12 \mathrm{~mm}$ lengths of $20 \mathrm{~cm}, 22 \mathrm{~cm}$, \&amp; $24 \mathrm{~cm}$. Cervical screws are of $8 \mathrm{~mm}$ cannulated with lengths varying from 70 to $110 \mathrm{~mm}$, Distal locking with two $5 \mathrm{~mm}$ screws. PFN for pertrochantric fractures is a versatile implant system which is used in both simple and complex fractures in the pertrochantric region. PFN consists of 3 components.1. The intramedullary nail.2. The cervical screws3. The distal locking screws. The cervical screws transmit the forces acting on the proximal femur to the intramedullary nail. Early load bearing of the fracture is possible. Two proximal screws ensure rotational stability of the PFN during the operation and subsequent healing process. The PFN is tapered at the distal end. This reduces stiffness and stress peaks, which can cause pain. Holes in the distal part allow a choice of either static or dynamic locking.

\section{Conclusion}

PFN is safe, effective and patient friendly device useful for the treatment of all intertrochanteric fractures irrespective of their comminution. Early mobilization and rehabilitation is possible since it is closed intramedullary procedure. Weight bearing should be delayed in severely comminuted fractures. It is mandatory to place both proximal screws in the neck, along with distal locking screws for better implant stability in osteoporotic as well as severely comminuted fractures.

\section{References}

1. Boyd HB, Griffin LL. Classification and treatment of trochanteric Fractures. Arch Surgery. 1944; 58:853-866.

2. Leung KS. 186 patients, 136 unstable fractures, Randomized Prospective study. JBJS. 1992; 784-B:34551.

3. Banan H, Al Sabti A, Jumulia T, Hart AJ. The treatment of unstable, extracapsular Hip fractures with the $\mathrm{AO} /$ ASIF Proximal Femoral Nail (PFN) - our first 60 cases. Injury. 2002; 33:401-405.

4. Aloyassari G, Langstaff RJ, Jones JWM, Al Lami. M, The AO/ ASIF, Proximal Femoral Nail (PFN) for the treatment of unstable trochanteric femoral fracture. Injury. 2002; 33:395-399.

5. Kyle Richard F, Cambell Sara J. Intertronchanteric fractures ", Chapter-40 Michael W. Chapman, Operative Orthopaedics. 1,600-603.

6. Nuber S, Ruter A. Stabilization for unstable trochanteric fractures by DHS/PFN Unfail chirurg. 2003; Jan; 1060) 3947. German

7. Suadan M, Lubbeke A, Sadonstai C, Riand N, Stem R, and Ho Hmeyer P. Petrochanteric fractures. Is there an advantage to an intramedullary nail? A randomized prospective study of 206 patients comparing DHS and PFN. J. Oms Trauma. 2002; 16(6):386-393.

8. Mahomed MN, Harrington IJ, Hearn TC Biomechanical analysis of the Medoff sliding plate". Trauma 2000; 48(1):93-100.

9. Parken MJ, Hondell HHG, Gamma, other cephalocondylic intramedullary nails Vs extra medullary implants for extra capsular hip fractures. Cochrane library. 2002, 3.

10. Ebraheim N, Mekhail A, Checroun A. Entry point of reconstruction nail, Am J Orthop. 1998; 27:474-476.

11. Seval B, Gavir JM, CE Gommo J, Doblave G. Finite element study of intramedullary osteosynthesis in the treatment of trochanteric fractures of Hip using Gamma nail and PFN. Injury. 2004; 35:130-135.

12. Rutt MS, Krikler SJ, Natie S, Ali MQ. Comparison of DHS and Gamma nail; a prospective, randomized controlled trial Injury. 1995; 26(9):615-618.

13. Simmermacher RK, J. Bos AM, Van Dev Werken. C. The AO/ ASIF - Proximal Femoral Nail (PFN); a new 
device for the treatment of unstable proximal femoral fractures. Injury. 1999; 30:327-332.

14. David A, Von Der Heyde D, Pommer A. Therapeutic Possibilities in Trochanteric fractures". Orthopade. 2000; 29(4):294-301.

15. Curter MJ, Jinnah RM, Wilson V. Cunningham. Proximal femoral fractures: A biomechanical study to compare intramedullary fixation. Injury. 1994; 25(2):99-104

16. Rosell PA, Parker MJ. Functional outcome after hip fractures. A 1 year prospective outcome study of 275 patients. Injury. 2003; 34:529-32.

17. Rubuzzi E, Pannonne A, Sehiavetti Santonello. D, de Nicalo V, Fancellu G. IMHS Clinical experience in the treatment of petrochanteric fractures. The results of a multicentric Italian study of 981 cases. Injury. 2002: 33:407-412. 